\title{
Reduced pro-inflammatory profile of $\gamma \delta T$ cells in pregnant patients with rheumatoid arthritis
}

Manuela Tham ${ }^{1,2}$, Gabriele R. Schlör ${ }^{1}$, Daniel Yerly ${ }^{1}$, Christina Mueller ${ }^{1}$, Daniel Surbek ${ }^{3}$, Peter M. Villiger ${ }^{1}$ and Frauke Förger ${ }^{1 *}$

\begin{abstract}
Background: During pregnancy, many patients with rheumatoid arthritis (RA) experience disease improvement, whereas patients with ankylosing spondylitis often suffer from persistent active disease. Here we investigated whether pregnancy-related changes in disease activity were associated with changes in the proportion and function of $\gamma \delta T$ cells.

Methods: The study population comprised 55 patients with RA, 31 patients with ankylosing spondylitis, and 35 healthy controls. Among these participants, 28 RA patients, 21 ankylosing spondylitis patients, and 23 healthy controls were investigated once before conception when possible, at each trimester of pregnancy, and at 8 weeks postpartum. Data were compared with age-matched non-pregnant patients to obtain disease-related background. In all subjects, peripheral V $\delta 1$ and V $\delta 2 T$ cells were analyzed for cell frequencies, the activation marker CD69, the cytotoxicity markers NKG2D and NKG2A, and the intracellular cytokines tumor necrosis factor (TNF)a, interferon $(\mathrm{IFN}) \mathrm{Y}$, interleukin (IL)-17 and IL-10.
\end{abstract}

Results: Pregnant patients showed a decreased V $\delta 2 N \delta 1$ ratio in the third trimester, which resulted from a slightly reduced proportion of V $\delta 2$ cells. Changes in RA disease activity during pregnancy and postpartum were not associated with numerical proportions of $\gamma \delta T$ cells but with changes of the cell activation marker CD69 on V $\delta 1$ and V $\delta 2$ cells. Only RA patients showed reduced proportions of TNFa-positive V $\delta 1$ and V $\delta 2$ cells and IFNY-positive V $\delta 2$ cells at the third trimester of pregnancy, a finding that was not apparent in the entire population of CD3 T cells. The proportions of IL-17-positive $\gamma \delta T$ cells and IL-10-positive $\gamma \delta T$ cells did not differ between pregnant and nonpregnant women of the different groups.

Conclusions: Changes of disease activity in pregnant RA patients were associated with functional changes in both $\gamma \delta T$ cell subsets. This reduced pro-inflammatory profile of $\gamma \delta T$ cells might contribute to the immunomodulation resulting in pregnancy-induced improvement of RA.

Keywords: Pregnancy, rheumatoid arthritis, ankylosing spondylitis, disease improvement, gammadelta T cells

\footnotetext{
* Correspondence: Frauke.Foerger@insel.ch

${ }^{1}$ Department of Rheumatology, Immunology and Allergology, University

Hospital and University of Bern, Bern, Switzerland

Full list of author information is available at the end of the article
} 


\section{Background}

Successful pregnancy requires an extraordinary state of natural immunomodulation to permit tolerance towards the haploidentical fetus [1]. The maternal immune system is altered to allow tolerance of fetal antigens while still being able to fight against infections. These immunomodulatory effects of pregnancy have varying influences on autoimmune rheumatic diseases. Rheumatoid arthritis (RA) improves in the majority of patients, whereas ankylosing spondylitis (AS) often remains active or is aggravated [2]. The understanding of relevant factors involved in the pregnancy-induced improvement of RA may enlarge our understanding of pathogenic factors involved in RA.

In this context, $\gamma \delta \mathrm{T}$ cells are of interest since they display immunoregulatory features during pregnancy [3]. These cells are unconventional $\mathrm{CD}^{+} \mathrm{T}$ cells that show features of both the innate and the adaptive immune system [4]. In contrast to $\alpha \beta \mathrm{T}$ cells (e.g., CD4 or CD8 T cells), $\gamma \delta \mathrm{T}$ cells recognize non-peptide antigens independently of classical major histocompatibility complex $(\mathrm{MHC})$ molecules [4]. $\gamma \delta \mathrm{T}$ cells exert their main functions through cytokine release, cytotoxicity, or antigen presentation [4]. In the peripheral blood of healthy humans, about $5 \%$ of lymphocytes express $\gamma \delta \mathrm{T}$ cell receptors [5]. Two main subsets of $\gamma \delta$ T cells bear different variable delta $(\mathrm{V} \delta)$ chains, $\mathrm{V} \delta 1$ and $\mathrm{V} \delta 2$, and show distinct tissue distributions and functions.

At the fetomaternal interface, $\gamma \delta$ T cells recognize fetusderived trophoblast cells that do not express polymorphic classical MHC class I molecules [6]. The pregnant uterus shows an increased number of $\gamma \delta \mathrm{T}$ cells [7]. V $\delta 1 \mathrm{~T}$ cells are the predominant subset in the decidua, where they produce interleukin (IL)-10 and transforming growth factor beta [8], while V $\delta 2 \mathrm{~T}$ cells predominate in the peripheral blood of healthy pregnant women $[9,10]$.

In autoimmune diseases, $\gamma \delta \mathrm{T}$ cells have shown both pro- and anti-inflammatory responses. In the collageninduced arthritis mouse model of RA, $\gamma \delta \mathrm{T}$ cells function differently at different disease stages, playing a proinflammatory role at arthritis onset and an antiinflammatory role once the disease is established [11]. Only very limited knowledge is available about $\gamma \delta \mathrm{T}$ cells in RA or AS in humans. One study reported reduced V $\delta 2$ $\mathrm{T}$ cells in the peripheral blood of RA patients compared to that of healthy controls [12]. Both V $\delta 1$ and $V \delta 2$ have been found in the synovium of RA patients with a predominance of interferon (IFN) $\gamma$-producing V $\delta 1$ cells $[13,14]$.

It is currently unknown whether $\gamma \delta \mathrm{T}$ cells play an immunoregulatory role in pregnancy-related amelioration of RA. We hypothesized that pregnancy has a systemic toleranceinducing effect that would shift the functional plasticity of circulating $\gamma \delta \mathrm{T}$ cells towards an anti-inflammatory profile, potentially impacting disease activity in RA. This shift would not be observed in a disease that remains active during pregnancy, such as AS. In the present study, we investigated phenotypical, and cytokine changes of circulating $\gamma \delta \mathrm{T}$ cells during pregnancy in patients with RA as compared to those with AS and healthy controls.

\section{Methods \\ Patients}

Here we prospectively studied 72 pregnant women: 28 with RA, 21 with AS, and 23 healthy controls. Pregnant individuals were examined once before conception when possible (up to 6 months before conception), once at each trimester (during gestational weeks 10-12, 20-22, and 30-32), and once at 8 weeks postpartum. Additionally, we studied 49 non-pregnant age-matched individuals, including 27 women with RA, 10 with AS, and 12 healthy women. Table 1 shows the patient characteristics and medications. All patients gave written informed consent, and the study was approved by the ethical committee of the Canton of Bern.

All patients were recruited from the pregnancy clinic of the Department of Rheumatology, Immunology, and Allergology and the Department of Obstetrics and Gynecology at the Inselspital of Bern, Switzerland. RA patients fulfilled the American College of Rheumatology criteria [15]. AS patients all had established axial involvement and fulfilled the modified New York Criteria [16]. RA disease activity was measured using the Disease Activity Score 28-C-reactive protein (DAS28-CRP) with three variables: swollen joint count, tender joint count, and C-reactive protein (CRP). AS disease activity was measured using the Ankylosing Spondylitis Disease Activity Score-C-reactive Protein (ASDAS-CRP). Serum CRP was measured either by Nycocard CRP Single Assay (Alere GmbH, Wädenswill, Switzerland) or highsensitivity CRP test (Department of Clinical Chemistry, Inselspital, University of Bern, Switzerland). The healthy control individuals included in the study each had a CRP below $5 \mathrm{mg} / \mathrm{L}$. Patients and healthy women with infections were excluded from the study.

\section{Cell preparation and flow cytometric analysis}

Peripheral blood mononuclear cells (PBMCs) were isolated from heparinized blood by standard density-gradient centrifugation over Biocoll (Biochrom AG, Berlin, Germany). For the frequency analysis of CD3, V $\delta 1$ and V82, and for the analysis of the activation marker and the cytotoxicity marker, the following directly labeled monoclonal antibodies were used: the PerCP-conjugated antibody CD3 (clone SK7) from Biolegend, the fluoresceinisothiocyanate-conjugated antibodies V $\delta 1$ (clone TS-1) and V82 (clone B6) from Thermo Scientific (Waltham, MA, USA), the phycoerythrin-coupled antibodies CD69 (clone FN50, Biolegend, San Diego, CA, USA), NKG2A (clone 
Table 1 Patient characteristics

\begin{tabular}{|c|c|c|c|}
\hline & $\begin{array}{l}\text { Healthy } \\
\text { control }\end{array}$ & $\begin{array}{l}\text { Rheumatoid } \\
\text { arthritis }\end{array}$ & $\begin{array}{l}\text { Ankylosing } \\
\text { spondylitis }\end{array}$ \\
\hline \multicolumn{4}{|l|}{ Total number } \\
\hline Total & 35 & 55 & 31 \\
\hline Non-pregnant & 12 & 27 & 10 \\
\hline Pregnant & 23 & 28 & 21 \\
\hline \multicolumn{4}{|c|}{ Age in years; median (range) } \\
\hline Non-pregnant & $30.5(24-48)$ & $32.5(17-51)$ & $35(30-47)$ \\
\hline Pregnant & $33(23-41)$ & $32(28-39)$ & $32.5(27-39)$ \\
\hline \multicolumn{4}{|c|}{ Rheumatoid factor positive } \\
\hline \multicolumn{2}{|l|}{ Non-pregnant } & $19(59.4)$ & \\
\hline \multicolumn{2}{|l|}{ Pregnant } & $13(46.4)$ & \\
\hline \multicolumn{4}{|l|}{ ACPA positive } \\
\hline \multicolumn{2}{|l|}{ Non-pregnant } & $17(53.1)$ & \\
\hline \multicolumn{2}{|l|}{ Pregnant } & $12(42.9)$ & \\
\hline \multicolumn{4}{|l|}{ HLA-B27 positive } \\
\hline \multicolumn{2}{|l|}{ Non-pregnant } & & $7(70)$ \\
\hline \multicolumn{2}{|l|}{ Pregnant } & & $14(66.7)$ \\
\hline \multicolumn{4}{|l|}{ Medication } \\
\hline \multicolumn{4}{|l|}{ NSAID } \\
\hline Non-pregnant & - & $25(75.8)$ & $7(70)$ \\
\hline $3^{\text {rd }}$ trimester & - & $4(14.3)$ & $5(23.8)$ \\
\hline Postpartum* & - & $7(25)$ & $9(42.9)$ \\
\hline \multicolumn{4}{|l|}{ Prednisone ${ }^{* *}$} \\
\hline Non-pregnant & - & $12(36.4)$ & $1(10)$ \\
\hline $3^{\text {rd }}$ trimester & - & $13(46.4)$ & $3(14.3)$ \\
\hline Postpartum* & - & $9(32.1)$ & $2(9.5)$ \\
\hline \multicolumn{4}{|l|}{ Sulfasalazine } \\
\hline Non-pregnant & - & $8(24.2)$ & $1(10)$ \\
\hline $3^{\text {rd }}$ trimester & - & $5(17.9)$ & - \\
\hline Postpartum* & - & $7(25)$ & $1(4.8)$ \\
\hline \multicolumn{4}{|l|}{ Antimalarials } \\
\hline Non-pregnant & - & $1(3)$ & - \\
\hline $3^{\text {rd }}$ trimester & - & $2(7.1)$ & - \\
\hline Postpartum* & - & $5(17.9)$ & - \\
\hline \multicolumn{4}{|l|}{ Methotrexate } \\
\hline Non-pregnant & - & $7(21.2)$ & - \\
\hline $3^{\text {rd }}$ trimester & - & - & - \\
\hline Postpartum* & - & - & - \\
\hline \multicolumn{4}{|l|}{ Leflunomide } \\
\hline Non-pregnant & - & $1(3)$ & - \\
\hline $3^{\text {rd }}$ trimester & - & - & - \\
\hline Postpartum* & - & - & - \\
\hline
\end{tabular}

Table 1 Patient characteristics (Continued)

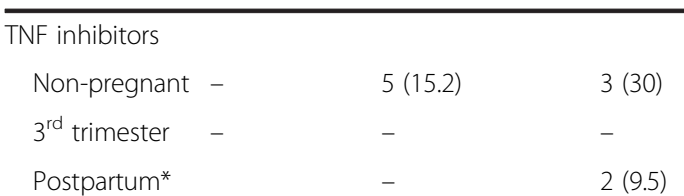

All values are shown as $n(\%)$ except where indicated otherwise. *Postpartum: 6-8 weeks after birth; ** $<15 \mathrm{mg} /$ day. ACPA anti citrullinated peptide antibodies, NSAID Non-steroidal anti-inflammatory drug (until gestation week 32), TNF Tumor necrosis factor

Z199, Beckman Coulter, Brea, CA, USA), and the allophycocyanin-coupled antibodies NKG2D (clone 1D11). Immunofluorescence staining was performed after washing the cells twice with phosphate-buffered saline containing $1 \%$ human serum. Cells were incubated for 20 minutes with each monoclonal antibody.

For the intracellular cytokine staining, cells were plated in 48-well plates at $1 \times 10^{6}$ cells $/ 100 \mu \mathrm{L}$ in complete RPMI 1640 containing $1 \times$ non-essential amino acids, $1 \times$ glutamine, $1 \times$ sodium pyruvate, $1 \times$ kanamycin (Life Technologies, Carlsbad, CA, USA), and $5 \%$ pooled human serum (Blood Transfusion Service, Bern, Switzerland) and stimulated with phorbol myristate acetate (PMA; $25 \mathrm{ng} / \mathrm{mL}$; Sigma Aldrich, St. Louis, MO, USA) and ionomycin ( $1 \mu \mathrm{g} / \mathrm{mL}$; Sigma Aldrich) for 4 hours in the presence of the protein transport inhibitor Brefeldin A (10 $\mu \mathrm{g} / \mathrm{mL}$; Sigma Aldrich). Intracellular cytokine staining was performed with the following antibodies: phycoerythrin-coupled antibodies, tumor necrosis factor (TNF) $\alpha$ (clone MAb11) and IL-10 (clone JES3-19 F1) from BD Biosciences (San Jose, CA, USA), and allophycocyanincoupled antibodies, IFNY (clone B27) from Biolegend and IL-17A (clone eBIO64DEC17) from eBioscience (San Diego, CA, USA). After surface and intracellular staining of PBMCs, data acquisition was performed using FACSCalibur 4-Color Cytometer (BD Biosciences), and data were analyzed using FlowJo Software (FlowJo, Ashland, OR, USA). Intracellular cytokine data were studied using both the proportion of cytokine-positive cells and the geometric mean fluorescence intensity (MFI) of a gated cell population.

\section{Statistical analysis}

All data are reported as the median and range. The Mann-Whitney $\mathrm{U}$ test was used for unpaired data analysis and group-wise comparison. The Wilcoxon signed-rank test was applied for longitudinal comparisons of paired samples. Linear regression analysis was used to investigate the association between disease activity and $\gamma \delta \mathrm{T}$ cell features. Data were analyzed using IBM SPSS Statistics 21 software. A $P$-value below 0.05 was considered significant.

\section{Results}

Disease activity improved in pregnant RA patients

A total of 49 pregnant patients and 23 healthy pregnant women were examined before (when possible), during 
and after pregnancy (Table 1). In RA patients, the disease activity scores according to DAS28-CRP were lower at the third trimester than 8 weeks postpartum $(P=0.03$; Fig. 1a). Though less medication was used by the pregnant patients than by the non-pregnant control group, inactive disease was commonly seen in pregnant RA patients, with 58-69 \% of RA patients showing low disease activity (DAS28-CRP scores <3.2; Fig. 1b). In contrast, disease activity in AS patients as measured by ASDASCRP remained unchanged during and after pregnancy (Fig. 1c). During pregnancy, $57-80 \%$ of AS patients experienced active disease (ASDAS-CRP scores $>2.1$; Fig. 1d).

\section{Discrete quantitative changes of $\mathrm{V} \delta 1$ and $\mathrm{V} \delta 2$ cells in pregnant patients}

With regard to the percentages of V $\delta 1$ and V $\delta 2 \mathrm{~T}$ cells within the $\mathrm{CD}^{+}$population, we first investigated the differences during and after pregnancy as well as the differences between pregnant and non-pregnant controls. Because of the lack of sufficient pre-pregnancy data in healthy women and AS patients, pre-pregnancy data were excluded from the analysis. In healthy subjects, the postpartum proportion of V $\delta 1 \mathrm{~T}$ cells was higher compared to that of the third trimester $(P=0.02)$ and higher compared to that of non-pregnant controls $(P=0.04$;
Fig. 2a). Concerning V $\delta 2$ cells of longitudinally followed healthy subjects, their frequency did not change. The proportion of $\mathrm{V} \delta 1$ cells and $\mathrm{V} \delta 2$ cells in pregnant healthy women was comparable to that of non-pregnant healthy women (Fig. 2a and b).

Among pregnant RA patients, V $\delta 1 \mathrm{~T}$ cell frequencies did not show significant changes during pregnancy. In contrast, V $22 \mathrm{~T}$ cells significantly decreased from the first trimester to the third trimester $(P=0.02)$, as well as from the second trimester to the third trimester $(P=0.02$; Fig. 2b). To detect small changes in the balance between $\mathrm{V} \delta 2$ and $\mathrm{V} \delta 1$ cells, we calculated the $\mathrm{V} \delta 2 / \mathrm{V} \delta 1$ ratio (Fig. 2c). In both pregnant RA patients and pregnant AS patients, the V $\delta 2 / \mathrm{V} \delta 1$ ratio decreased from the first trimester to the third trimester (RA, $P=0.01$; AS, $P=0.03$ ). Among pregnant AS patients, the $\mathrm{V} \delta 2 / \mathrm{V} \delta 1$ ratio was lower at the third trimester than at the postpartum examination $(P=0.04)$. These changes in the $\mathrm{V} \delta 2 / \mathrm{V} \delta 1$ ratio could not be observed in healthy controls.

Overall, only discrete quantitative changes of $V \delta 1$ and V82 T cells were observed during pregnancy, which were more pronounced in cases of RA than in AS. Interestingly, V82 T cells decreased during pregnancy in cases of RA when disease activity was very low. In contrast, nonpregnant RA patients with active disease (DAS28-CRP
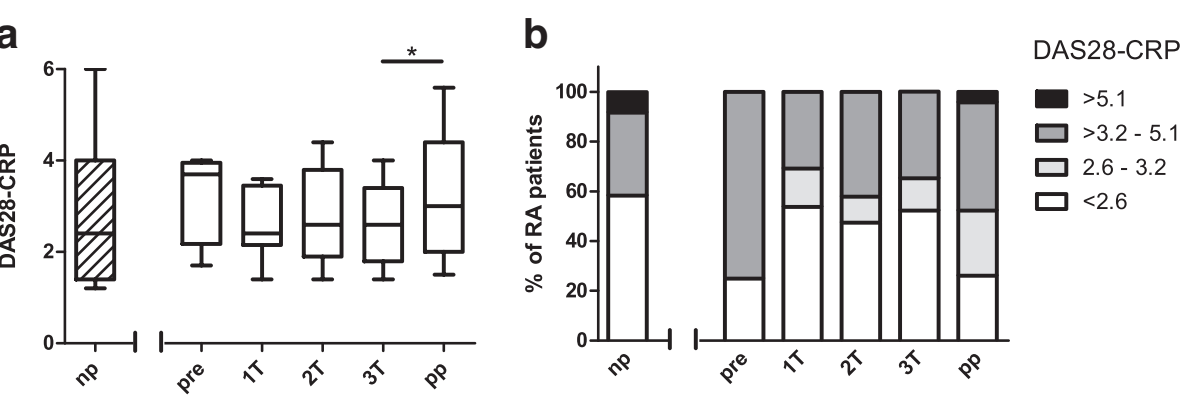

C

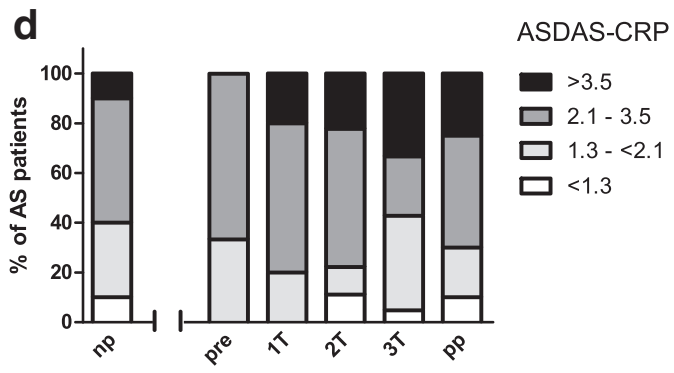

Fig. 1 Disease activity before, during and after pregnancy. a Disease activity measured by the Disease Activity Score in 28 joints and C-reactive protein (DAS28-CRP) for 28 longitudinally followed pregnant rheumatoid arthritis (RA) patients and for 27 non-pregnant RA patients. b The graph shows the percentages of the abovementioned patients grouped according to DAS28-CRP scores into: remission (DAS28-CRP $<2.6$ ), low disease activity (DAS 28-CRP 2.6-3.2), moderate disease activity (DAS28-CRP > 3.2-5.1) and high disease activity (DAS28-CRP $>5.1$ ). c Disease activity measured by the Ankylosing Spondylitis Disease Activity Score and C-reactive protein (ASDAS-CRP) for 21 longitudinally followed pregnant ankylosing spondylitis (AS) patients and for 10 non-pregnant $(n p)$ AS patients. $\mathbf{d}$ The graph shows the percentages of patients grouped according to ASDAS-CRP scores into: inactive disease (ASDAS <1.3), moderate disease activity (ASDAS 1.3-<2.1), high disease activity (ASDAS 2.1-3.5) and very high disease activity (ASDAS >3.5). Pregnant patients were analyzed once before pregnancy (pre), at each trimester (1T, 2T, 3T) and 8 weeks postpartum (pp). Boxplots show the median and the interquartile ranges. ${ }^{*} P<0.05$ 


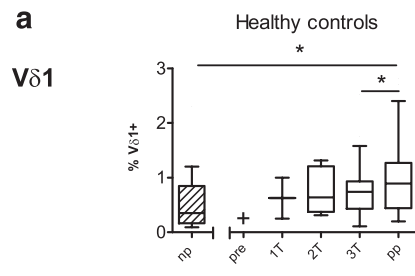

b

$\mathbf{V} \delta 2$

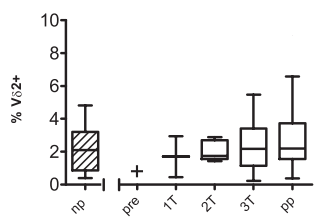

c

$\mathrm{V} \delta 2 / \mathrm{V} \delta 1$

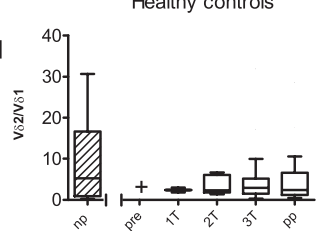

Rheumatoid arthritis

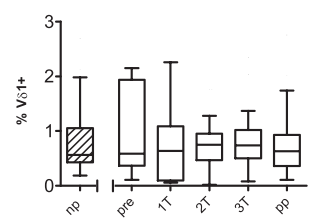

Rheumatoid arthritis

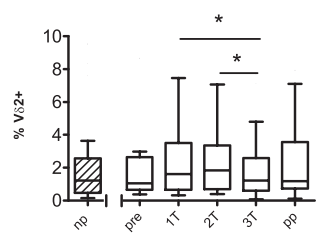

Rheumatoid arthritis

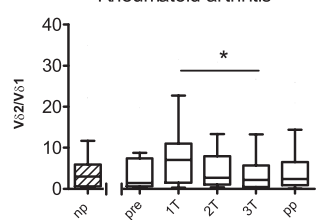

Ankylosing spondylitis

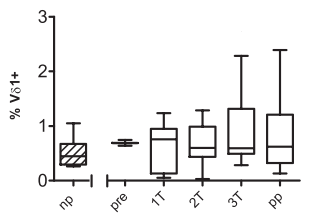

Ankylosing spondylitis
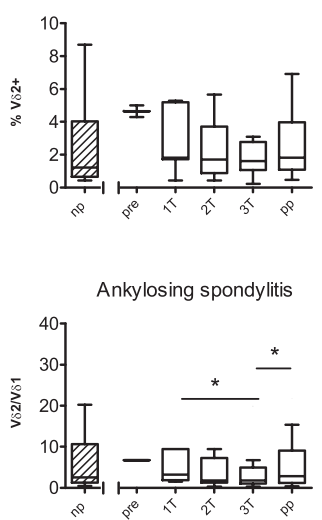

Fig. 2 Longitudinal changes of $V \delta 1$ and $V \delta 2 T$ cells during and after pregnancy. The proportions of $V \delta 1$ (a) and $V \delta 2$ cells (b) and the V $\delta 2 N \delta 1$ ratio (c) are shown for healthy controls (left panels), patients with RA (middle panels) and patients with AS (right panels). Pregnant individuals were studied before pregnancy (pre), at each trimester $(1 T, 2 T, 3 T)$ and postpartum $(p p)$. Values were compared with age-matched non-pregnant ( $n p)$ individuals in each group. Frequencies of $\mathrm{V} \delta 1$ and $V \delta 2 \mathrm{~T}$ cells are shown as percentages of CD3-positive T cells in isolated PBMCs. Boxplots show the median and the interquartile ranges. + no data available. ${ }^{*} P<0.05$

$>3.2$ ) showed a lower frequency of $\mathrm{V} \delta 2 \mathrm{~T}$ cells (median 0.80 , range $0.16-3.48$ ) compared to non-pregnant RA patients with the inactive disease (median 2.04, range 0.24-9.54; $P=0.04$; data not shown).

\section{Association between disease activity and activation marker expressed by $\gamma \delta \mathrm{T}$ cells of RA patients}

To investigate whether pregnancy-related changes of disease activity were reflected by changes of cell activation, we analysed the expression of the activation marker CD69 on V $\delta 1$ and V $\delta 2$ T cells. The proportion of CD69positive V $\delta 1$ and V $\delta 2$ cells did not differ between nonpregnant healthy women and those at the third trimester or postpartum time point (Fig. 3a and b). By contrast, RA patients analyzed 8 weeks postpartum showed higher percentages of CD69-positive V $\delta 1$ cells than nonpregnant controls $(P=0.01$; Fig. 3a). In AS patients, the proportions of CD69-positive V82 were highest at the third trimester and differed from those of non-pregnant AS patients $(P=0.02)$ and from those of postpartum AS patients $(P=0.04)$. The relationship between disease activity and cell activation of $\gamma \delta \mathrm{T}$ cells was then analysed by linear regression analysis in non-pregnant, pregnant (third trimester) and postpartum patients. In RA patients, disease activity as measured by DAS28-CRP correlated with the proportion of CD69-expressing V $\delta 1$ and V82 cells (Fig. 3c). In AS patients, the association of CD69-bearing $\mathrm{V} \delta 2$ cells and disease activity measured by ASDAS-CRP showed a slight tendency towards statistical significance ( $P=0.08$; Fig. $3 \mathrm{~d})$. There was no significant association between CD69-bearing V $\delta 1$ cells and ASDAS-CRP.

\section{Increased expression of anti-cytotoxic markers on $\gamma \delta T$ cells of pregnant RA patients}

The cytotoxic response of $\gamma \delta$ T cells is modulated by the activating natural killer (NK) cell receptor NKG2D and by the inhibiting NK cell receptor NKG2A. We profiled $\gamma \delta \mathrm{T}$ cells for NKG2A and NKG2D expressions to investigate whether pregnancy changed the balance between these functionally different receptors. The proportions of NKG2D-expressing V $\delta 1$ and $V \delta 2$ cells did not change from the first trimester until the postpartum time-point in any group (data not shown). Similar results were seen when analyzing the MFI of NKG2D among the population of $\mathrm{V} \delta 1$ and $\mathrm{V} \delta 2$ cells.

Pregnant RA patients only displayed a longitudinal change of NKG2A-positive V $\delta 1 \mathrm{~T}$ cells, with an approximately 2.4-fold higher proportion at the second trimester than at the postpartum time-point (median percentage of NKG2A-positive cells: second trimester, 7.53; postpartum, 3.04; $P=0.02$; data not shown). This difference was not 


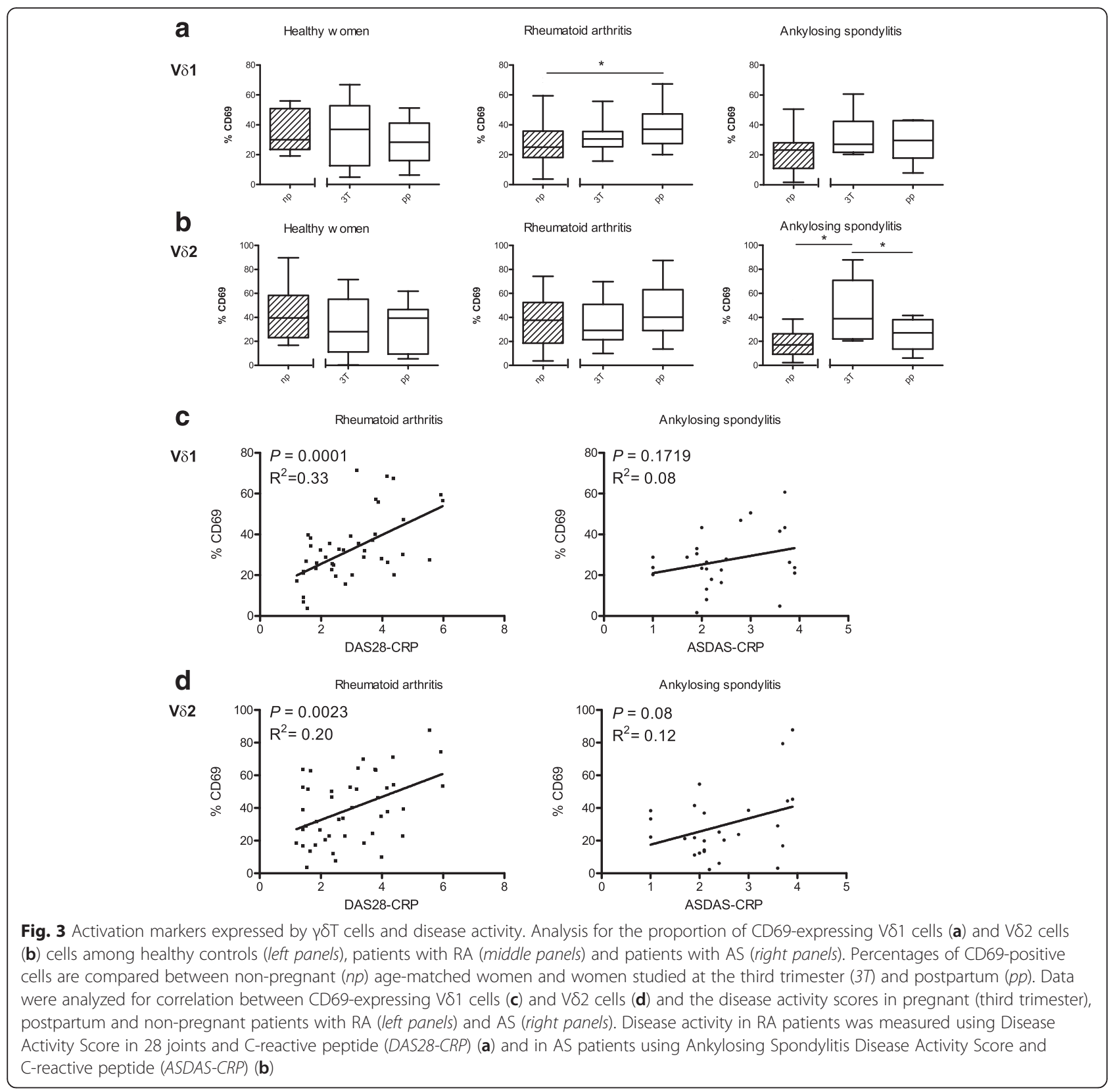

seen when analyzing the MFI of NKG2A among the population of V $\delta 1$ cells. The proportion of NKG2A-expressing $\mathrm{V} \delta 2$ cells was stable during and after pregnancy in all groups.

\section{Reduced TNFa and IFN $\gamma$ production by $\gamma \delta T$ cells in pregnant RA patients}

To determine the intracellular cytokine profile of $\gamma \delta \mathrm{T}$ cells, freshly isolated PBMCs were activated with PMA and ionomycin in the presence of a Golgi transport inhibitor and stained for TNF $\alpha$, IFN $\gamma$, IL-17 and IL-10. We first analyzed longitudinal changes of cytokine- positive V $\delta 1$ and $V \delta 2$ cells in healthy women and patients. In RA patients, the population of TNF $\alpha$-producing $\mathrm{V} \delta 1$ cells decreased from the second to the third trimester and increased from the third trimester to the postpartum time point (second to third trimester analyzed by percentage of positive cells and by MFI: $P=0.04$; third trimester to postpartum analyzed by MFI: $P=0.04$; Additional file 1 : Figure S1).

We then compared differences between pregnant and non-pregnant individuals. For pregnant individuals, the third trimester was chosen since this time point was related to clinical changes of disease activity and 


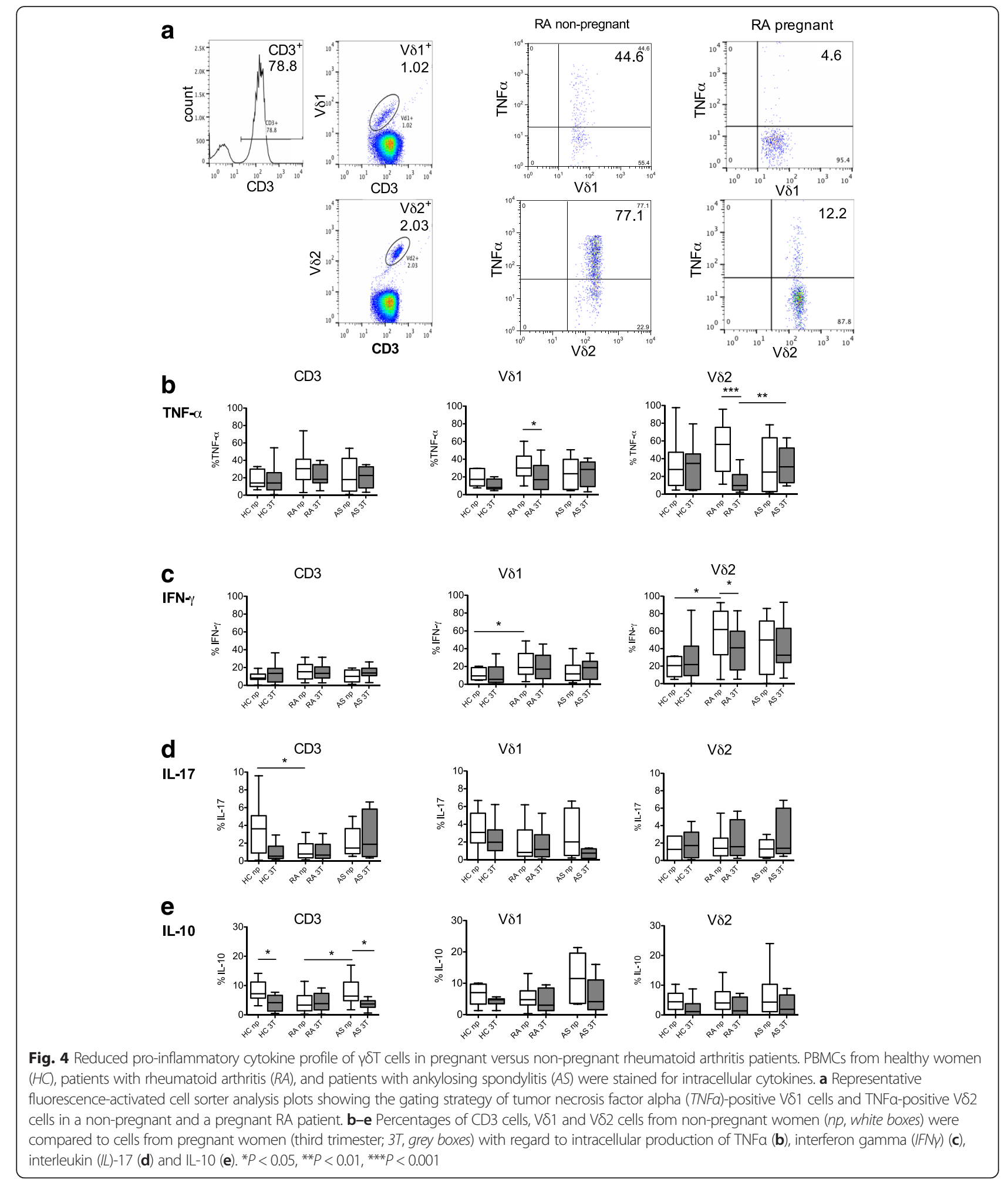

quantitative changes of $\gamma \delta$ T cells. Regarding TNF $\alpha$ production, the most pronounced difference appeared between pregnant and non-pregnant RA patients (Fig. 4a and b). Pregnant RA patients showed a lower proportion of TNF $\alpha$-positive V $\delta 1$ cells compared to non-pregnant
RA patients $(P=0.04)$. Similarly, pregnant RA patients showed an approximately 5.7-fold lower proportion of TNF $\alpha$-positive V $\delta 2$ cells compared to non-pregnant RA patients $(P=0.001)$. With respect to IFN $\gamma$ (Fig. $4 \mathrm{c})$, nonpregnant RA patients displayed the highest proportions 
of IFN $\gamma$-positive $\mathrm{V} \delta 1$ and IFN $\gamma$-positive $\mathrm{V} \delta 2$ cells, which significantly differed from the median proportions found in non-pregnant healthy controls (V $\delta 1, P=0.04$; $\mathrm{V} \delta 2, P=0.02)$. Analogous to the TNF $\alpha$ results, the proportion of IFN $\gamma$-positive V $\delta 2$ cells was lower in pregnant RA patients compared to non-pregnant RA patients $(P=0.03$; Fig. $4 c)$. Similar results were found when analyzing the MFI of TNF $\alpha$ and IFN $\gamma$ among the population of $\mathrm{V} \delta 1$ and $V \delta 2$ cells (Additional file 2: Figure S2). Interestingly, the reduced percentages of TNF $\alpha$ - and IFN $\gamma$-positive $\gamma \delta \mathrm{T}$ cells observed in pregnant RA patients were not apparent in the entire population of $\mathrm{CD} 3 \mathrm{~T}$ cells (Fig. $4 \mathrm{~b}$ and c, left panels).

With respect to IL-17, we found no significant difference in the proportion of IL-17-positive V $\delta 1$ or $V \delta 2$ cells between pregnant and non-pregnant women (Fig. $4 \mathrm{~d}$ ). The median proportions of IL-17-positive V $\delta 1$ and V $\delta 2$ cells were below $4 \%$. Regarding IL-10, IL-10-positive cells tended to be lower in pregnant than in nonpregnant individuals for $\mathrm{V} \delta 1$ and $\mathrm{V} \delta 2 \mathrm{~T}$ cells, an effect which was more pronounced for the population of CD3 cells (Fig. 4e). There was no influence of medication on the proportion of cytokine-positive V $\delta 1$ or $V \delta 2$ cells in non-pregnant patients.

Overall, among all analyzed individuals, only pregnant RA patients showed a reduced production of proinflammatory cytokines by V $\delta 1$ and V $\delta 2$ cells.

\section{Discussion}

Pregnancy has a positive effect on RA, but has no beneficial effect on AS. In the present study, we analyzed whether pregnancy-related improvements of RA symptoms were associated with changes of circulating $\gamma \delta \mathrm{T}$ cells. $\gamma \delta \mathrm{T}$ cells are an immune system component that bridge the innate and the adaptive immune response, and they show a functional plasticity ranging from immunoregulatory properties to pro-inflammatory responses. We hypothesized that pregnancy has not only a local- but also a systemic tolerance-inducing effect on immunoregulatory cells such as $\gamma \delta \mathrm{T}$ cells, which might support pregnancy-induced amelioration of RA.

Overall, our results demonstrate a reduced proinflammatory cytokine profile of $\gamma \delta \mathrm{T}$ cells in pregnant patients with RA compared to non-pregnant RA patients. This functional modification of $\gamma \delta \mathrm{T}$ cells occurred in pregnant RA patients of whom $69 \%$ had low disease activity but not in patients with AS, a disease with ongoing disease activity.

Among the two subsets of circulating $\gamma \delta \mathrm{T}$ cells, changes were detected in both the V $\delta 1$ and the $V \delta 2$ cells. Against our expectation based on previous findings on V $\delta 1$ and V $\delta 2$ cells, we did not find an increase in circulating V $\delta 1$ cells during pregnancy that provide a Th2 cytokine pattern as shown for decidual V $\delta 1$ cells $[8,10]$. This discrepancy may be explained by the analysis of peripheral V $\delta 1$ and $V \delta 2$ cells and the use of different sampling periods. Among pregnant patients with RA and $\mathrm{AS}$, we identified reduced $\mathrm{V} \delta 2 / \mathrm{V} \delta 1$ ratios during the third trimester of pregnancy, which were due to a slight pregnancy-related reduction of $V \delta 2$ cells. However, a previous study also reported reduced V $\delta 2$ cell percentages among non-pregnant RA patients, a finding that was not related to disease activity assessed by CRP and erythrocyte sedimentation rate [17]. By contrast, our cohort of non-pregnant RA patients showed a lower frequency of $\mathrm{V} \delta 2 \mathrm{~T}$ cells in cases with active disease (DAS28-CRP >3.2) compared to cases with inactive disease (DAS28-CRP <3.2). Similarly, patients with juvenile idiopathic arthritis (JIA) suffering from more active disease showed lower percentages of synovial $V \delta 1$ and $V \delta 2$ $\mathrm{T}$ cells [18]. Therefore, we suppose that V $\delta 2 \mathrm{~T}$ cell frequency variations can be triggered by pregnancy and by disease activity in RA.

However, these quantitative changes may be less important than functional changes of $\gamma \delta \mathrm{T}$ cells in terms of reflecting disease activity. In our cohort of pregnant and non-pregnant RA patients, disease activity was associated with $V \delta 1$ and $V \delta 2 T$ cells that were positive for the activation marker CD69. Similarly, high CD69 expression on V82 cells has been reported in inflamed joints of JIA patients [19]. However, in our RA patients, disease activity correlated with CD69 positivity on both V $\delta 1$ and V $\delta 2$ cells, which do not respond to the same antigen. Thus, the increased percentage of CD69-positive V $\delta 1$ and V $\delta 2$ cells might be induced by a disease-related inflammatory environment rather than by specific antigens.

$\gamma \delta$ T cells exert their effector function via both cytokine release and cytotoxicity. Cytotoxic potency has been described for both V $\delta 1$ and V $\delta 2$ cells [20, 21], along with modulation by the activating NK cell receptor NKG2D or the inhibiting NK cell receptor NKG2A. The placenta secretes NKG2D ligands, which could downregulate NKG2D [22]. Therefore, we expected to find a pregnancy-induced change of the balance between the NKG2D and NKG2A expressions on V $\delta 1$ and V $\delta 2$ cells. However, our results showed that the percentages of NKG2D-expressing V $\delta 1$ and V $\delta 2$ cells remained unchanged during pregnancy and postpartum. In contrast, the percentage of NKG2A-expressing V $\delta 1$ cells was higher during the second trimester than postpartum in RA patients. A previous report demonstrated a pregnancyrelated increase of NKG2A-positive $\gamma \delta \mathrm{T}$ cells in healthy women analyzed around gestational week 27 compared to non-pregnant controls [10]. The increased expression of NKG2A on $\gamma \delta$ T cells might therefore be a pregnancyrelated phenomenon. In this respect, one might speculate that trophoblast-derived HLA-E binds to the increased 
proportion of NKG2A-positive $\gamma \delta \mathrm{T}$ cells, thereby downmodulating their cytotoxic potential [23, 24].

Given that $\gamma \delta \mathrm{T}$ cells can produce the pro-inflammatory cytokines TNF $\alpha$ and IFN $\gamma$, which are involved in the pathogenesis of RA and spondyloarthritis [25, 26], it is possible that pregnancy induces some dampening of the TNF $\alpha$ - or IFN $\gamma$-producing V $\delta 1$ and V $\delta 2$ cells. This speculation was indeed supported by results in RA patients, but not in AS patients or in healthy controls. Therefore, an effect of pregnancy alone, which would appear in all groups, seems unlikely. However, the local cytokine environment has a decisive role in driving $\gamma \delta \mathrm{T}$ cell function in a way that normal pregnancy gives rise to $\gamma \delta \mathrm{T}$ cells with an antiinflammatory profile whereas pre-eclampsia gives rise to $\gamma \delta \mathrm{T}$ cells with a pro-inflammatory profile $[27,28]$. The anti-inflammatory cytokine milieu created by gestational $\gamma \delta \mathrm{T}$ cells in turn promotes feto-maternal tolerance [28]. Thus, the downregulation of TNF $\alpha$ - and IFN $\gamma$-positive $\gamma \delta \mathrm{T}$ cells in pregnant versus non-pregnant RA patients could result from a reduced inflammatory milieu that in turn supports disease improvement.

Disease activity in AS remained unchanged during pregnancy. Accordingly, there was no change of TNF $\alpha$ - and IFN $\gamma$-positive $\gamma \delta$ T cells in pregnant versus non-pregnant AS patients. Previous research in AS has shown that a $\gamma \delta \mathrm{T}$ cell subpopulation bearing the IL-23 receptor is the main source of IL-17-secreting T cells [29]. Our analysis showed similar percentages of IL-17-secreting $\gamma \delta \mathrm{T}$ cells, with no differences between the study groups or between pregnant and non-pregnant individuals. Thus, the persistent active disease present in pregnant AS patients provides a pro-inflammatory milieu that might overrule the regulatory features of $\gamma \delta \mathrm{T}$ cells.

The limitations of our study are the limited and unequal number of patients and controls, and the scarce pre-pregnancy and first trimester data of healthy pregnant women. Differences in the frequency and function of $\gamma \delta \mathrm{T}$ cells might be missed in early pregnancy of healthy controls. However, we assume that age-matched, non-pregnant healthy women would not differ from healthy women examined prior to conception.

\section{Conclusions}

In conclusion, here we observed functional changes of $\gamma \delta \mathrm{T}$ cells that are associated with disease activity in a cohort of pregnant versus non-pregnant RA patients. These functional changes of $\gamma \delta$ T cells in pregnant RA patients which were mainly reflected by decreases of TNF $\alpha$ - and IFN $\gamma$-positive $\gamma \delta$ T cells seem to be a consequence of an anti-inflammatory environment. No such changes of $\gamma \delta \mathrm{T}$ cells were seen in AS, a disease that remains active during pregnancy. The more tolerogenic profile of $\gamma \delta \mathrm{T}$ cells in pregnant RA patients might contribute to the process of immunomodulation in pregnancy.

\section{Additional files}

Additional file 1: Figure S1. Longitudinal changes of TNFa-producing $\gamma \delta T$ cells in pregnant RA patients. Patients with rheumatoid arthritis were analyzed for the percentages (A) and the mean fluorescence intensities (MFI) (B) of TNFa-producing CD3 cells (left panel), V 81 cells (middle panel) and V $\delta 2$ cells (right panel) before pregnancy (pre), at each trimester (1T, 2T, 3T) and postpartum (pp). Values are expressed as median and interquartile ranges. ${ }^{*} P<0.05$. (PDF $33 \mathrm{~kb}$ )

Additional file 2: Figure S2. Reduced pro-inflammatory cytokine profile of $\gamma \delta T$ cells in pregnant versus non-pregnant RA patients. Peripheral blood mononuclear cells from healthy women $(\mathrm{HC})$, patients with rheumatoid arthritis (RA), and patients with ankylosing spondylitis (AS) were stained for intracellular cytokines. Mean fluorescence intensity (MFI) of TNFa (A), IFNY (B), IL-17 (C) and IL-10 (D) among CD3 cells (left panels), V $\delta 1$ cells (middle panels) and V $\delta 2$ cells (right panels) from non-pregnant (np, white boxes) and pregnant women (third trimester, 3T, grey boxes) were analyzed. Box plots show the median and the interquartile ranges. ${ }^{*} P<0.05,{ }^{* *} P<0.01,{ }^{* * *} P<0.001$. (PDF $55 \mathrm{~kb}$ )

\section{Abbreviations}

ACPA: Anti citrullinated peptide antibodies; AS: Ankylosing spondylitis; ASDAD-CRP: Ankylosing Spondylitis Disease Activity Score-C-reactive Protein; CD: Cluster of differentiation; CRP: C-reactive protein; DAS28-CRP: Disease Activity Score 28-C-reactive protein; IFN: Interferon; IL: Interleukin; JIA: Juvenile idiopathic arthritis; MFI: Mean fluorescence intensity; MHC: Major

histocompatibility complex; NK: Natural killer; PBMC: Peripheral blood mononuclear cell; PMA: Phorbol myristate acetate; RA: Rheumatoid arthritis; TNF: Tumor necrosis factor; V§: Variable delta.

\section{Competing interests}

The authors declare that they have no competing interests.

\section{Authors' contributions}

MT carried out the sample collection, study execution, and analysis, and drafted the manuscript. GRS and CM were involved in data acquisition of non-pregnant individuals and revision of the manuscript. DS was involved in sample collection, data interpretation and manuscript revising. DY was involved in data analysis, data interpretation, and revision of the manuscript. PMV was involved in study design, data interpretation and manuscript drafting. FF was involved in study design, data analysis and interpretation, drafting of the article and its final edition. All authors read and approved the final manuscript.

\section{Acknowledgements}

This work was supported by grants from the Käthe-Zingg-Schwichtenberg Fonds (KZS 18/09), the Olga-Mayenfisch Fonds, and the AbbVie Rheumatology Grant 2009.

\section{Author details}

${ }^{1}$ Department of Rheumatology, Immunology and Allergology, University Hospital and University of Bern, Bern, Switzerland. ' Graduate School, University of Bern, Bern, Switzerland. ${ }^{3}$ Department of Obstetrics and Gynecology, University Hospital and University of Bern, Bern, Switzerland.

Received: 26 May 2015 Accepted: 6 January 2016

Published online: 22 January 2016

\section{References}

1. Arck PC, Hecher K. Fetomaternal immune cross-talk and its consequences for maternal and offspring's health. Nat Med. 2013;19(5): 548-56. doi:10.1038/nm.3160.

2. Ostensen $M$, Villiger $P M$, Forger $F$. Interaction of pregnancy and autoimmune rheumatic disease. Autoimmun Rev. 2012;11(6-7):A437-46. doi:10.1016/j.autrev.2011.11.013.

3. Mincheva-Nilsson L, Hammarstrom S, Hammarstrom ML. Human decidual leukocytes from early pregnancy contain high numbers of gamma delta + cells and show selective down-regulation of alloreactivity. J Immunol. 1992;149(6): 2203-11.

4. Hayday AC. Gammadelta T, cells and the lymphoid stress-surveillance response. Immunity. 2009;31(2):184-96. doi:10.1016/j.immuni.2009.08.006. 
5. Carding SR, Egan PJ. Gammadelta T cells: functional plasticity and heterogeneity. Nat Rev Immunol. 2002;2(5):336-45. doi:10.1038/nri797.

6. Toussirot E, Saas P, Deschamps M, Pouthier F, Perrot $L$, Perruche $S$, et al. Increased production of soluble CTLA-4 in patients with spondylarthropathies correlates with disease activity. Arthritis Res Ther. 2009; 11(4):R101. doi:10.1186/ar2747.

7. Kimura M, Hanawa H, Watanabe H, Ogawa M, Abo T. Synchronous expansion of intermediate TCR cells in the liver and uterus during pregnancy. Cell Immunol. 1995;162(1):16-25. doi:10.1006/cimm.1995.1046.

8. Nagaeva O, Jonsson L, Mincheva-Nilsson L. Dominant IL-10 and TGF-beta mRNA expression in gammadeltaT cells of human early pregnancy decidua suggests immunoregulatory potential. Am J Reprod Immunol. 2002;48(1):9-17.

9. Barakonyi A, Polgar B, Szekeres-Bartho J. The role of gamma/delta T-cell receptorpositive cells in pregnancy: part II. Am J Reprod Immunol. 1999;42(2):83-7.

10. Szereday L, Barakonyi A, Miko E, Varga P, Szekeres-Bartho J. Gamma/deltaTcell subsets, NKG2A expression and apoptosis of Vdelta2+ T cells in pregnant women with or without risk of premature pregnancy termination. Am J Reprod Immunol. 2003;50(6):490-6.

11. Peterman GM, Spencer C, Sperling Al, Bluestone JA. Role of gamma delta T cells in murine collagen-induced arthritis. J Immunol. 1993;151(11):6546-58.

12. Smith MD, Broker B, Moretta L, Ciccone E, Grossi CE, Edwards JC, et al. T gamma delta cells and their subsets in blood and synovial tissue from rheumatoid arthritis patients. Scand J Immunol. 1990;32(6):585-93.

13. Ito $Y$, Usui $T$, Kobayashi $S$, Iguchi-Hashimoto $M$, Ito $H$, Yoshitomi H, et al. Gamma/delta T cells are the predominant source of interleukin-17 in affected joints in collagen-induced arthritis, but not in rheumatoid arthritis. Arthritis Rheum. 2009;60(8):2294-303. doi:10.1002/art.24687.

14. Shen Y, Li S, Quayle AJ, Mellbye OJ, Natvig JB, Forre O. TCR gamma/delta + cell subsets in the synovial membranes of patients with rheumatoid arthritis and juvenile rheumatoid arthritis. Scand J Immunol. 1992;36(4):533-40.

15. Arnett FC, Edworthy SM, Bloch DA, McShane DJ, Fries JF, Cooper NS, et al. The American Rheumatism Association 1987 revised criteria for the classification of rheumatoid arthritis. Arthritis Rheum. 1988;31(3):315-24.

16. van der Linden S, Valkenburg HA, Cats A. Evaluation of diagnostic criteria for ankylosing spondylitis. A proposal for modification of the New York criteria. Arthritis Rheum. 1984;27(4):361-8.

17. Lunardi C, Marguerie C, Walport MJ, So AK. T gamma delta cells and their subsets in blood and synovial fluid from patients with rheumatoid arthritis. Br J Rheumatol. 1992;31(8):527-30.

18. Berkun Y, Bendersky A, Gerstein M, Goldstein I, Padeh S, Bank I. GammadeltaT cells in juvenile idiopathic arthritis: higher percentages of synovial Vdelta1+ and Vgamma9+ T cell subsets are associated with milder disease. J Rheumatol. 2011;38(6):1123-9. doi:10.3899/jrheum.100938.

19. Bendersky A, Marcu-Malina V, Berkun Y, Gerstein M, Nagar M, Goldstein I, et al. Cellular interactions of synovial fluid gammadelta T cells in juvenile idiopathic arthritis. J Immunol. 2012;188(9):4349-59. doi:10.4049/jimmunol.1102403.

20. Mincheva-Nilsson L, Nagaeva O, Sundqvist KG, Hammarstrom ML, Hammarstrom S, Baranov V. gammadelta T cells of human early pregnancy decidua: evidence for cytotoxic potency. Int Immunol. 2000;12(5):585-96.

21. Roessner K, Wolfe J, Shi C, Sigal LH, Huber S, Budd RC. High expression of Fas ligand by synovial fluid-derived gamma delta T cells in Lyme arthritis. Immunol. 2003;170(5):2702-10.

22. Hedlund M, Stenqvist AC, Nagaeva O, Kjellberg L, Wulff M, Baranov V, et al. Human placenta expresses and secretes NKG2D ligands via exosomes that down-modulate the cognate receptor expression: evidence for immunosuppressive function. J Immunol. 2009;183(1):340-51. doi:10.4049/ jimmunol.0803477.

23. Barakonyi A, Kovacs KT, Miko E, Szereday L, Varga P, Szekeres-Bartho J. Recognition of nonclassical HLA class I antigens by gamma delta T cells during pregnancy. J Immunol. 2002;168(6):2683-8.

24. Ishitani A, Sageshima N, Hatake K. The involvement of HLA-E and $-\mathrm{F}$ in pregnancy. J Reprod Immunol. 2006;69(2):101-13. doi:10.1016/j.jri.2005. 10.004.

25. Hu C, Qian L, Miao Y, Huang Q, Miao P, Wang P, et al. Antigen-presenting effects of effector memory Vgamma9Vdelta2 T cells in rheumatoid arthritis. Cell Mol Immunol. 2012;9(3):245-54. doi:10.1038/cmi.2011.50.

26. Bonneville M, O'Brien RL, Born WK. Gammadelta T cell effector functions: a blend of innate programming and acquired plasticity. Nat Rev Immunol. 2010;10(7):467-78. doi:10.1038/nri2781.

27. Miko E, Szereday L, Barakonyi A, Jarkovich A, Varga P, Szekeres-Bartho J. Immunoactivation in preeclampsia: Vdelta2+ and regulatory $T$ cells during the inflammatory stage of disease. J Reprod Immunol. 2009;80(1-2):100-8. doi:10.1016/j.jri.2009.01.003.

28. Exley MA, Boyson JE. Protective role of regulatory decidual gammadelta T cells in pregnancy. Clin Immunol. 2011;141(3):236-9. doi:10.1016/j.clim.2011.09.004

29. Kenna TJ, Davidson SI, Duan R, Bradbury LA, McFarlane J, Smith M, et al. Enrichment of circulating interleukin-17-secreting interleukin-23 receptorpositive gamma/delta T cells in patients with active ankylosing spondylitis. Arthritis Rheum. 2012;64(5):1420-9. doi:10.1002/art.33507.

\section{Submit your next manuscript to BioMed Central and we will help you at every step:}

- We accept pre-submission inquiries

- Our selector tool helps you to find the most relevant journal

- We provide round the clock customer support

- Convenient online submission

- Thorough peer review

- Inclusion in PubMed and all major indexing services

- Maximum visibility for your research

Submit your manuscript at www.biomedcentral.com/submit
) Biomed Central 